\title{
(2) OPEN ACCESS \\ One-year myopia control efficacy of spectacle lenses with aspherical lenslets
}

\author{
Jinhua Bao, ${ }^{1,2}$ Adeline Yang, ${ }^{2,3}$ Yingying Huang $(0), 1,2$ Xue Li, ${ }^{1,2}$ Yiguo Pan, ${ }^{1,2}$ \\ Chenglu Ding, ${ }^{1,2}$ Ee Woon Lim, ${ }^{2,3}$ Jingwei Zheng, ${ }^{1}$ Daniel P Spiegel, ${ }^{2,3}$ Björn Drobe, ${ }^{2,3}$ \\ Fan Lu (10), ${ }^{1,2}$ Hao Chen (ib) ${ }^{1,2}$
}

- Additional material is published online only. To view please visit the journal online (http://dx.doi.org/10.1136/ bjophthalmol-2020-318367).

${ }^{1}$ Eye Hospital and School of Ophthalmology and Optometry, Wenzhou Medical University, Wenzhou, Zhejiang, China Wenzhou Medical UniversityEssilor International Research Center (WEIRC), Wenzhou Medical University, Wenzhou, Zhejiang, China

${ }^{3}$ R\&D AMERA, Essilor International, Singapore

\section{Correspondence to} Professor Hao Chen and Professor Fan Lu, Eye Hospital and School of Ophthalmology and Optometry, Wenzhou Medical University, Wenzhou, China; chenhao@mail.eye.ac.cn, Lufan62@mail.eye.ac.cn

$\mathrm{JB}$ and $\mathrm{AY}$ contributed equally.

Received 9 November 2020 Revised 28 February 2021 Accepted 1 March 2021

\section{ABSTRACT}

Aims To evaluate the 1-year efficacy of two new myopia control spectacle lenses with lenslets of different asphericity.

Methods One hundred seventy schoolchildren aged 8-13 years with myopia of $-0.75 \mathrm{D}$ to $-4.75 \mathrm{D}$ were randomised to receive spectacle lenses with highly aspherical lenslets (HAL), spectacle lenses with slightly aspherical lenslets (SAL), or single-vision spectacle lenses (SVL). Cycloplegic autorefraction (spherical equivalent refraction (SER)), axial length $(A L)$ and best-corrected visual acuity (BCVA) were measured at baseline and 6-month intervals. Adaptation and compliance questionnaires were administered during all visits. Results After 1 year, the mean changes in the SER $( \pm$ SE) and $A L( \pm S E)$ in the SVL group were $-0.81 \pm 0.06 \mathrm{D}$ and $0.36 \pm 0.02 \mathrm{~mm}$. Compared with SVL, the myopia control efficacy measured using SER was $67 \%$ (difference of 0.53 D) for HAL and $41 \%$ (difference of $0.33 \mathrm{D}$ ) for $\mathrm{SAL}$, and the efficacy measured using AL was $64 \%$ (difference of $0.23 \mathrm{~mm}$ ) for HAL and $31 \%$ (difference of $0.11 \mathrm{~mm}$ ) for SAL (all $p<0.01$ ). HAL resulted in significantly greater myopia control than SAL for SER (difference of $0.21 \mathrm{D}$, $p<0.001$ ) and $A L$ (difference of $0.12 \mathrm{~mm}, p<0.001$ ). The mean BCVA $(-0.01 \pm 0.1 \log M A R, p=0.22)$ and mean daily wearing time $(13.2 \pm 2.6$ hours, $p=0.26)$ were similar among the three groups. All groups adapted to their lenses with no reported adverse events, complaints or discomfort.

Conclusions Spectacle lenses with aspherical lenslets effectively slow myopia progression and axial elongation compared with SVL. Myopia control efficacy increased with lenslet asphericity.

Trial registration number ChiCTR1800017683.

\section{INTRODUCTION}

The prevalence of myopia has been increasing in East Asia and other parts of the world, with rates of myopia and high myopia projected to reach approximately $50 \%$ and $10 \%$ of the global population, respectively, in $2050 .^{1}$ With uncorrected myopia incurring about US\$244 billion in 2015, the economic impact of this myopia epidemic will increase substantially, ${ }^{2}$ as well as the risk of myopia-related pathologies, such as glaucoma, myopic macular degeneration and retinal detachment. $^{34}$ The use of myopia control interventions ${ }^{5}$ can help reduce severity of myopia, which potentially diminishes the risk of these pathologies. ${ }^{34}$
Research in animal models has shown that numerous properties of optical defocus, such as sign, degree and retinal distribution, have substantial effects on eye growth. The sign of the imposed optical defocus, that is, whether the focal plane is in front of or behind the retina, has a different effect on eye growth. Myopic defocus tends to slow eye growth, whereas hyperopic defocus drives eye elongation, ${ }^{67}$ and the larger the amount of defocus the stronger effect on eye growth. ${ }^{8}$ Observations from animal studies also showed that when the eye was presented with equal amounts of competing defocus, myopic defocus produced a stronger effect than hyperopic defocus, resulting in slower eye growth. ${ }^{910}$

Two other studies in animals investigated the effect of aspherical lenses with a power gradient on emmetropisation. ${ }^{11} 12$ Instead of focusing light on two distinct surfaces, as in the case of competing defocus lenses, these aspherical lenses deviate rays of light continuously in a nonlinear manner that creates a three-dimensional quantity of light in front of the retina, which we call volume of myopic defocus (VoMD) in this paper. Greater asphericity, that is, a larger VoMD, reduces lens-induced myopia in chicks. ${ }^{11}{ }^{12}$ Many of the findings from animal studies have been used to design myopia control interventions in humans, such as specific spectacle lenses ${ }^{13} 14$ and contact lenses. ${ }^{1516}$

Building on previous findings and based on optical modelling, we tested two different spectacle lens designs for myopia control that induce two different VoMD through two types of aspherical lenslets (figure 1). The purpose of this article is to: (1) compare 1-year change in spherical equivalent of cycloplegic autorefraction (SER) and axial length (AL) between single-vision spectacle lenses (SVL) and two spectacle lenses with different VoMD values based on optical modelling, (2) to test the hypothesis that aspherical lenslets slow myopia progression in a dose-dependent manner and (3) to report the initial outcome of best-corrected visual acuity (BCVA), adaptation and compliance of using lenses with aspherical lenslets.

\section{MATERIALS AND METHODS Study oversight}

This study started in July 2018 and is ongoing at the Eye Hospital of Wenzhou Medical University. A data and safety monitoring committee (DSMC) 


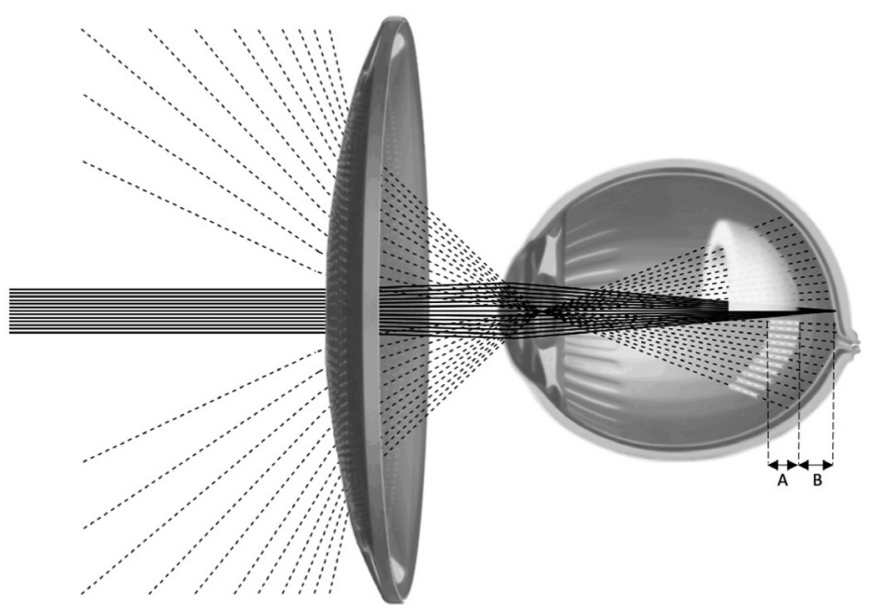

Figure 1 Illustration of the study device providing a volume of myopic defocus (VoMD) (white shell) in front of the retina through 11 concentric rings of contiguous lenslets ( $A=$ depth of VoMD and $B=$ distance from the retina). The calculations for the lenslets were based on the modified Atchison eye model ${ }^{32}$ using a retinal shape modified to match the peripheral refraction data of Chinese children. ${ }^{33-35}$ Spectacle lenses with highly aspherical lenslets $(A=0.7 \mathrm{~mm}$ and $B=1.2 \mathrm{~mm})$, spectacle lenses with slightly aspherical lenslets $(A=0.3 \mathrm{~mm}$ and $B=1.0$ $\mathrm{mm}$ ) (illustrated and authorised by Dr. Damien Paillé from R\&D AMERA, Essilor International).

oversees the trial and reviews the trial data for patient safety at regular intervals.

\section{Study design}

This 2-year clinical trial was designed to be a randomised, controlled, double-masked study with follow-up visits every 6 months and a planned interim analysis after 12 months by the DSMC. Potentially eligible children were referred from the hospital to attend a screening visit. The following inclusion criteria were used: cycloplegic SER between -0.75 $\mathrm{D}$ and $-4.75 \mathrm{D}$; astigmatism of cycloplegic autorefraction not exceeding $1.50 \mathrm{D}$; anisometropia not exceeding $1.00 \mathrm{D}$ based on SER; monocular BCVA of $0.05 \log$ MAR or better at distance for both eyes; absence of ocular pathology; absence of binocular vision issues; and no history of ocular surgery or use of myopia control measures. Eligible participants enrolled in the study were assigned to receive spectacle lenses with highly aspherical lenslets (HAL), spectacle lenses with slightly aspherical lenslets (SAL), or SVL in a 1:1:1 ratio using adaptive randomisation with online software (www.rando.la) to balance treatment groups based on baseline right-eye refractive error, mean age and gender. Masked examiners performed cycloplegic autorefraction and AL measurements. Spectacles were not labelled to identify the treatment group assigned. Identifiable data were removed with only outcome variables and treatment group provided to the DSMC for interim analysis by the study manager in charge of lens logistics.

\section{Interventions}

All spectacle lenses were made of polycarbonate. HAL and SAL treatment spectacle lenses have a spherical front surface with 11 concentric rings formed by contiguous aspherical lenslets (diameter of $1.1 \mathrm{~mm}$ ). The area of the lens without lenslets provides distance correction. The geometry of aspherical lenslets has been calculated to generate a VoMD in front of the retina at any eccentricity, serving as a myopia control signal (figure 1).

\section{Outcome variables}

The objective ophthalmic parameters collected at each visit were SER and AL before dispensing. Baseline measures, such as near horizontal phoria (33 cm, modified Thorington technique), lag of accommodation ( $33 \mathrm{~cm}$, calculated using equations ${ }^{17}$ ), distance and near BCVA using best-corrected study device, were collected after dispensing (online supplemental methods). The main outcome variables were changes in SER and AL from baseline. SER (sphere plus half-cylinder of the mode of 10 measurements using a Topcon KR-800, Topcon Corporation, Japan) was measured at least $30 \mathrm{~min}$ after instillation of two drops of $1 \%$ cyclopentolate administered $5 \mathrm{~min}$ apart. AL was measured by calculating the average of five measurements obtained using a Lenstar LS900 instrument (Haag-Streit AG, Switzerland).

The secondary outcome variables include BCVA during dispensing visits, time needed to adapt to the lenses (no reported complaints or discomfort) and compliance (self-reported daily wearing hours per week) during 6-month follow-up visits. Distance BCVA using manifest refraction with study device was measured using a multifunctional VA tester (MFVA-100; BriteEye Medical Tech Co., Shenzhen, China) ${ }^{18}$ under $80 \mathrm{~cd} / \mathrm{m}^{2}$ at a distance of $5.5 \mathrm{~m}$. Near BCVA was measured using $100 \%$ contrast ETDRS (Precision Vision, USA) near chart at $40 \mathrm{~cm}$ under 200 lux (online supplemental methods). The examiners administered a questionnaire to the participants during 6-month and 12-month visits to assess the wearing hours during each 6-month period for compliance and duration to adapt to the lenses with feedback provided for adaptation (online supplemental eTable 2). Average daily wearing hours for each 6-month period was based on self-report of the participant. The average daily wearing hours were calculated based on the total daily wearing time in a week in the 6 -month period: ( 6 -month weekly total +12 -month weekly total)/2 periods/7 days). A phone interview was also conducted 3 days and 2 weeks after dispensing to ensure and record adaptation outcomes (online supplemental eTable 1). Adaptation was reported based on phone interview and 6-month self-responded questionnaires. Adaptation was defined as wearing the study device with no discomfort, problems and decrease in visual acuity.

\section{Sample size}

The minimum sample size was 150 , based on projections of a $33 \%$ reduction in the amount of SER and AL progression for treatment groups compared with control group and a mean SER progression of $1.50 \mathrm{D}$ with a SD of $0.75 \mathrm{D}$ and converted $\mathrm{AL}$ progression of $0.6 \mathrm{~mm}$ with a SD of $0.02 \mathrm{~mm}$ after 2 years in the control group based on previous findings. ${ }^{1920}$ This was based on a two-sided statistical test with a 1\% type I error threshold, $90 \%$ power and a $10 \%$ drop-out rate. ${ }^{19} 21$

\section{Statistical analysis}

All data from patients who completed 1-year follow-up were analysed. The mean values for ocular parameters measured in the right eye were used, as no significant differences in changes in SER (mean difference of $-0.006 \mathrm{D}, \mathrm{p}=0.82$; correlation between eyes, $r=0.71, p<0.001$ ) and AL (mean difference of $0.01 \mathrm{~mm}, \mathrm{p}=0.19$; correlation between eyes, $\mathrm{r}=0.84, \mathrm{p}<0.001$ ) were observed between eyes.

The change in parameters was defined as the difference between baseline and corresponding follow-up measurements. 


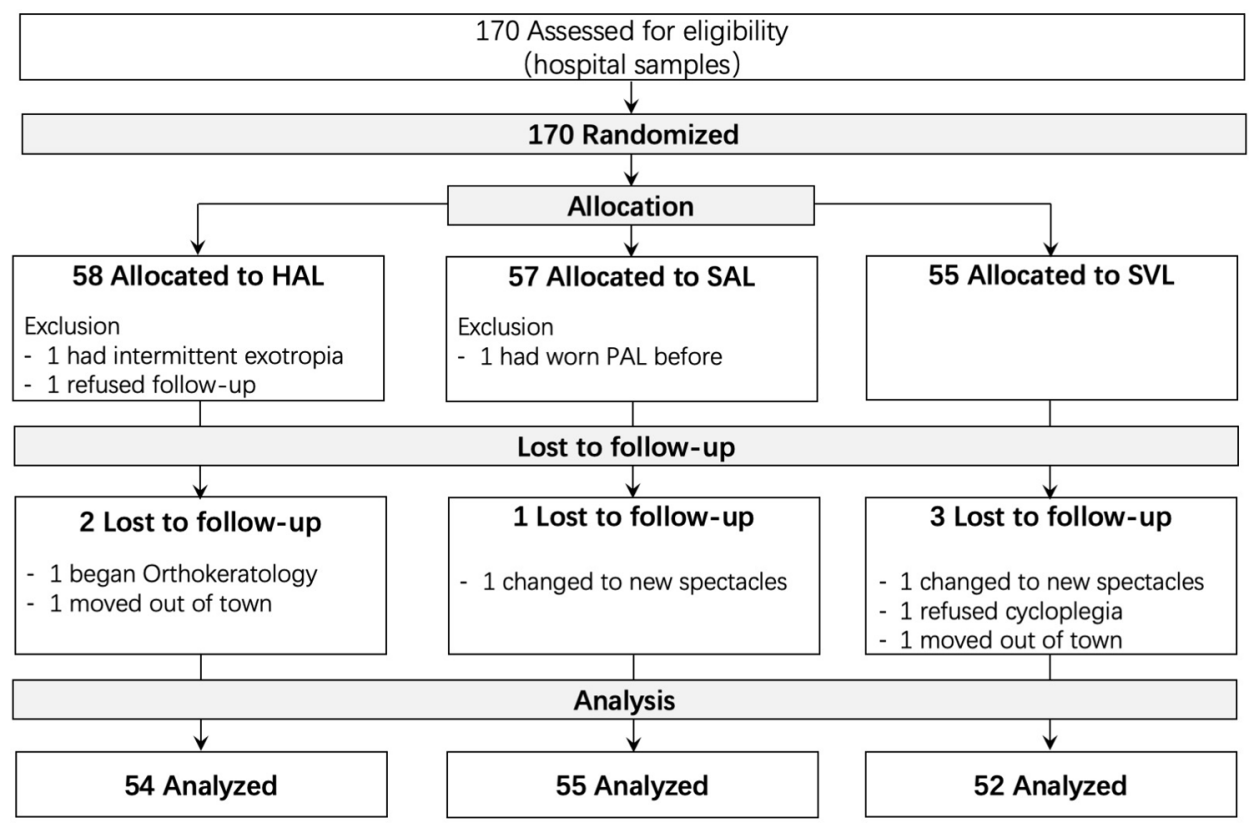

Figure 2 Consolidated Standards of Reporting Trials flow chart of the study, showing participant randomisation, treatment group assignment, follow-up visits and data analysis. (Two participants switched to new spectacles because they wanted trendier frames instead of those provided by the study.) HAL, spectacle lenses with highly aspherical lenslets; PAL, progressive addition lenses; SAL, spectacle lenses with slightly aspherical lenslets; SVL, single-vision spectacle lenses.

The $\chi^{2}$ test and analysis of variance with post hoc Bonferroni test were used to assess intergroup differences in categorical and continuous variables, respectively. Our analysis was performed using complete case data without imputation for missing data and dropouts. We performed analyses using a linear mixed model, adjusted for baseline age, gender, SER, AL, age of myopia onset and the number of parents with myopia to evaluate the treatment effect. IBM SPSS Statistics for Windows, V.24.0 (IBM Corp. Released 2016, Armonk, New York, USA: IBM Corp), was used for data analysis. Two-sided p values of less than 0.05 were considered as statistically significant. The difference between treatment groups was adjusted using step-down Bonferroni method.

\section{RESULTS}

\section{Study population}

One hundred seventy children with myopia and a mean $( \pm S D)$ age of $10.4 \pm 1.2$ years, range $8-13$ years old, were referred from the hospital based on the inclusion criteria and randomised among the HAL $(n=58)$, SAL $(n=57)$ and SVL $(n=55$; figure 2$)$ groups. The SAL group included a higher proportion of girls and shorter AL than the other groups (table 1).

Of the 170 randomised children, only 167 were dispensed with the study equipment. Three children discontinued: one had intermittent exotropia that was not apparent during screening, one belatedly reported a history of using myopia control and one dropped out. At the 1-year visit, 161 participants had completed their visits, whereas six participants did not; the participants who did not attend the follow-up comprised two (3.6\%), one $(1.8 \%)$ and three $(5.5 \%)$ participants from the HAL, SAL and SVL groups, respectively (figure 2). Reasons for drop-out were not related to the study device.

\section{Changes in SER}

Table 2 presents the mean $( \pm \mathrm{SE}$ ) changes in myopia (1 year) for 161 participants randomised to one of three groups in the study. Significant differences were observed among the treatment groups $(\mathrm{F}(2,158)=20.58, \mathrm{p}<0.001)$. Both the HAL and SAL groups exhibited less SER progression (by $0.53 \mathrm{D}$ and 0.33 $\mathrm{D}$, respectively; both $\mathrm{p}<0.001$ ) than the SVL group. In addition, the HAL group displayed less SER progression than the SAL group by a difference of $0.21 \mathrm{D}(\mathrm{p}=0.04$; table 2$)$.

In the linear mixed model analysis, baseline age was significantly associated with SER progression $(p=0.02)$. The model-adjusted mean changes in SER were $-0.30 \pm 0.06 \mathrm{D}$, $-0.48 \pm 0.06 \mathrm{D}$ and $-0.79 \pm 0.06 \mathrm{D}$ for the HAL, SAL and SVL groups, respectively (figure 3), with a significant effect of lens design $(\mathrm{F}(2,154.45)=18.94, \mathrm{p}<0.001)$. Compared with

Table 1 Baseline characteristics of participants who completed the 12-month follow-up in each treatment group

\begin{tabular}{|c|c|c|c|}
\hline & $\begin{array}{l}\text { HAL } \\
(n=54)\end{array}$ & $\begin{array}{l}\text { SAL } \\
(n=55)\end{array}$ & $\begin{array}{l}\text { SVL } \\
(n=52)\end{array}$ \\
\hline Age (years) & $10.7 \pm 0.2$ & $10.1 \pm 0.2$ & $10.4 \pm 0.2$ \\
\hline \multicolumn{4}{|l|}{ Gender } \\
\hline Male, $\%$ (n) & $48(26)$ & $33(18)$ & $56(29)$ \\
\hline Cycloplegic SER (D) & $-2.70 \pm 0.14$ & $-2.31 \pm 0.13$ & $-2.46 \pm 0.12$ \\
\hline $\mathrm{AL}(\mathrm{mm})$ & $24.76 \pm 0.09$ & $24.43 \pm 0.10$ & $24.77 \pm 0.09$ \\
\hline${ }^{*}$ Near phoria $(\Delta)$ & $-2.36 \pm 0.90$ & $-2.24 \pm 0.88$ & $-1.86 \pm 0.92$ \\
\hline${ }^{*}$ Accommodative lag at $33 \mathrm{~cm}$ (D) & $0.94 \pm 0.05$ & $1.09 \pm 0.04$ & $1.03 \pm 0.05$ \\
\hline Age of myopia onset (years) & $9.3 \pm 0.2$ & $9.3 \pm 0.2$ & $9.4 \pm 0.2$ \\
\hline \multicolumn{4}{|l|}{ Myopic parents, \% (n) } \\
\hline 0 & $33(18)$ & $22(12)$ & $23(12)$ \\
\hline 1 & $37(20)$ & $42(23)$ & 37 (19) \\
\hline 2 & $30(16)$ & $36(20)$ & $40(21)$ \\
\hline
\end{tabular}

Data are presented as the means $\pm \mathrm{SEs}$, unless stated otherwise.

* Measured using the best corrected study device after dispensing.

$\triangle$, prism diopters; $A L$, axial length; $D$, diopters; $H A L$, spectacle lenses with highly aspherical lenslets; SAL, spectacle lenses with slightly aspherical lenslets; SER, spherical equivalent refraction; SVL, single-vision spectacle lenses. 


\begin{tabular}{|c|c|c|c|c|}
\hline & $\begin{array}{l}\text { HAL } \\
(n=54)\end{array}$ & $\begin{array}{l}\text { SAL } \\
(n=55)\end{array}$ & $\begin{array}{l}\text { SVL } \\
(n=52)\end{array}$ & $P$ value \\
\hline \multicolumn{5}{|c|}{ Cycloplegic SER (D) } \\
\hline 6 months & $-0.10 \pm 0.04$ & $-0.17 \pm 0.04$ & $-0.34 \pm 0.04$ & $<0.001$ * \\
\hline 12 months & $-0.27 \pm 0.06$ & $-0.48 \pm 0.05$ & $-0.81 \pm 0.06$ & $<0.001$ * \\
\hline \multicolumn{5}{|l|}{$\mathrm{AL}(\mathrm{mm})$} \\
\hline 6 months & $0.08 \pm 0.01$ & $0.14 \pm 0.01$ & $0.20 \pm 0.01$ & $<0.001^{*}$ \\
\hline 12 months & $0.13 \pm 0.02$ & $0.25 \pm 0.02$ & $0.36 \pm 0.02$ & $<0.001$ * \\
\hline $\begin{array}{l}\text { Data are presen } \\
{ }^{*} \text { The differences } \\
\text { statistically sign } \\
\text { AL, axial length; } \\
\text { aspherical lensle }\end{array}$ & $\begin{array}{l}\text { means } \pm \text { SEs. } \\
\text { s in cycloplegic } \\
\text { ferent at } 6 \text { and } \\
; \text { HAL, spectac } \\
\text { herical equival }\end{array}$ & $\begin{array}{l}\text { ve error and ax } \\
\text { ths. } \\
\text { with highly as }\end{array}$ & $\begin{array}{l}\text { on between tre } \\
\text { slets; SAL, spec } \\
\text { ectacle lenses. }\end{array}$ & $\begin{array}{l}\text { uns were all } \\
\text { s with slightly }\end{array}$ \\
\hline
\end{tabular}

SVL, the adjusted differences in mean SER were 0.50 D (63\%, $\mathrm{p}<0.001)$ and $0.32 \mathrm{D}(40 \%, \mathrm{p}<0.001)$ in the HAL and SAL groups, respectively. Pearson's correlation analyses showed that age $(r=-0.43, p=0.002)$ was negatively correlated with the changes in SER (faster progression in younger participants) only in the SVL group, but not in the HAL $(r=-0.18, p=0.20)$ and SAL $(r=-0.22, p=0.11)$ groups.

\section{Changes in $\mathrm{AL}$}

Table 2 presents the mean $( \pm \mathrm{SE})$ unadjusted increases in $\mathrm{AL}(1$ year) for 161 participants randomised to one of three groups in the study. Similar to SER, a significant difference $(\mathrm{F}(2$, $158)=26.50, \mathrm{p}<0.001)$ was observed among the treatment groups. Compared with SVL, HAL and SAL reduced AL elongation by $0.23 \mathrm{~mm}$ and $0.11 \mathrm{~mm}$, respectively (both $\mathrm{p}<0.001$; table 2). Furthermore, HAL resulted in less AL elongation than SAL by $0.12 \mathrm{~mm}(\mathrm{p}=0.001)$.

In the linear mixed model analysis, baseline age $(p=0.001)$ and age of myopia onset $(p=0.01)$ were significantly associated with AL elongation. After adjustment, the mean changes in AL were $0.14 \pm 0.02 \mathrm{~mm}, 0.24 \pm 0.02 \mathrm{~mm}$ and $0.35 \pm 0.02 \mathrm{~mm}$ for participants wearing HAL, SAL and SVL, respectively, with $61 \%(0.21$ $\mathrm{mm}, \mathrm{p}<0.001)$ and $31 \%(0.11 \mathrm{~mm}, \mathrm{p}=0.001)$ reductions in AL elongation in the HAL and SAL groups compared with the SVL group (figure 3 ). HAL produced less $\mathrm{AL}$ elongation than SAL by $0.11 \mathrm{~mm}(43 \%, \mathrm{p}=0.001)$. A younger age ( $\mathrm{SVL}, \mathrm{r}=-0.55$, $\mathrm{p}<0.001$; SAL, $\mathrm{r}=-0.47, \mathrm{p}<0.001)$ and earlier age of myopia onset (SVL, $\mathrm{r}=-0.38, \mathrm{p}=0.005$; SAL, $\mathrm{r}=-0.33, \mathrm{p}=0.01$ ) were correlated with greater AL changes in the SVL and SAL groups, but not in the HAL group.
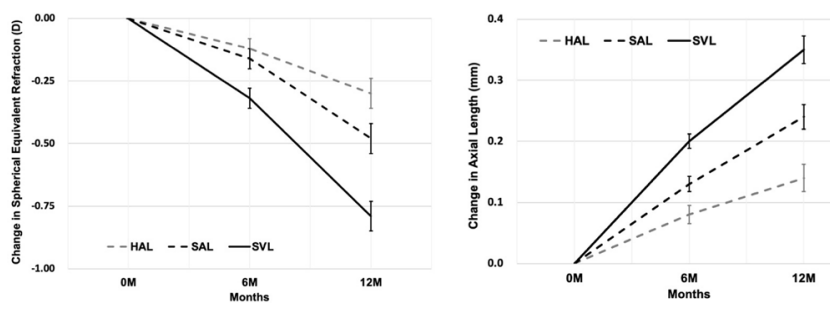

Figure 3 Adjusted mean change from baseline spherical equivalent refraction (left panel) and axial length (right panel) in each treatment group over a 1-year period. Error bars represent $1 \mathrm{SE}$ of the mean. 0 $\mathrm{M}=0$-month baseline; $6 \mathrm{M}=6$-month follow-up; $12 \mathrm{M}=12$ month followup. HAL, spectacle lenses with highly aspherical lenslets; SAL, spectacle lenses with slightly aspherical lenslets; SVL, single-vision spectacle lenses.

\section{Distribution of participants with myopia progression}

Twenty per cent of participants in the HAL group, $4 \%$ in the SAL group and none in the SVL group experienced a hyperopic shift (decrease of myopia). The percentages of participants without changes in SER were 8\% in the HAL group, 7\% in the SAL group and $2 \%$ in the SVL group. Notably, $72 \%$ of participants in the HAL group, $89 \%$ in the SAL group and $98 \%$ in the SVL group experienced a myopic shift (online supplemental eFigure 1). The percentages of participants who did not display an increase in AL were 28\% in the HAL group, 9\% in the SAL group and $0 \%$ in the SVL group. A decrease in AL was observed in $26 \%$ of participants in the HAL group, $5 \%$ in the SAL group and $0 \%$ in the SVL group (online supplemental eFigure 1).

\section{Visual performance, compliance and adaptation}

Distance BCVA did not differ significantly among HAL, SAL and SVL groups $(0.01 \pm 0.02 \log \mathrm{MAR},-0.02 \pm 0.01 \log \mathrm{MAR}$ and $-0.02 \pm 0.01$ logMAR, respectively; $F=1.52, p=0.22)$, and no difference was observed in near BCVA $(0.15 \pm 0.01$ $\log$ MAR, $0.14 \pm 0.01 \log$ MAR and $0.12 \pm 0.01 \log M A R$, respectively; $\mathrm{F}=2.07, \mathrm{p}=0.13$ ). No differences in near horizontal phoria $(p=0.92)$ and the lag of accommodation $(p=0.07)$ were observed between the HAL, SAL and SVL groups (table 1).

Daily wearing time was similar among the treatment groups, with mean durations of $12.9 \pm 0.36$ hours, $13.6 \pm 0.32$ hours and $13.1 \pm 0.36$ hours for participants in the HAL, SAL and SVL groups, respectively $(F(2,158)=1.06, p=0.35)$. No significant difference was observed in the proportion of participants who adapted to the spectacle lenses within 3 days among the HAL, SAL and SVL groups $(90 \%, 100 \%$ and 94\%, respectively; $\mathrm{p}=0.07)$. All treatment groups were adapted to study devices within a week and had no complaints or discomfort, based on the phone interview and 6-month questionnaires. Adverse events (untoward medical occurrence, unintended disease or injury or any untoward clinical signs related to the interventions) were not reported (online supplemental methods).

\section{DISCUSSION}

HAL and SAL were effective at controlling myopia progression, and HAL was more effective at controlling myopia progression than SAL. BCVA, adaptation and compliance were not affected by the lens design.

Previous studies in chicks showed that an aspherical lens with a greater power gradient reduces lens-induced myopia. ${ }^{11} 12$ Additionally, the amount of myopic defocus was directly linked to the induced experimental hyperopia under competing defocus paradigm. $^{922} \mathrm{HAL}$ induced a larger VoMD in front of the retina than SAL based on optical modelling; hence, we hypothesise that a larger VoMD will be more effective at controlling myopia progression. Further studies are required to elucidate the role of the size and position of VoMD and their combination in the efficacy of myopia control.

In our study, a younger age was associated with faster myopia progression in the SVL group and faster AL elongation in the SVL and SAL groups, similar to previous myopia control trials. ${ }^{1423}$ However, this trend was not observed in children wearing HAL, that is, changes in SER and AL in this treatment group were similar across all age groups. Given the increased risk of high myopia with a younger age of myopia onset, ${ }^{24} 25$ HAL might represent a promising intervention for controlling myopia during early childhood to slow myopia progression and reduce myopia-related risks later in life. 
A significant hyperopic shift was observed in $20 \%$ of participants wearing HAL and $4 \%$ of participants wearing SAL. A reduction in AL was also observed in $26 \%$ of participants in the HAL group and $5 \%$ in the SAL group. In contrast, these changes were absent in the SVL group. Overall, 15\% of participants wearing HAL and $2 \%$ of participants wearing SAL showed a hyperopic shift and a reduction in AL. This phenomenon of a hyperopic shift due to lens compensation has been shown in several animal models ${ }^{672627}$ but rarely reported in human intervention studies. Although the underlying mechanism remains unknown, the human eye can detect the presence and profile of optical defocus to undergo compensatory changes in AL. ${ }^{5} \mathrm{~A}$ short-term decrease in AL was reported in two human studies, likely through an increase in choroidal thickness. ${ }^{28} 29$ However, the reported increase (approximately $0.03 \mathrm{~mm}$ ) was not clinically significant. Choroidal thickening may be linked to myopia regression in our study, but other potential factors, including the retina and sclera, also influence changes in AL. ${ }^{3031}$ Further investigations of choroidal thickness are ongoing in this study.

The current study reports the first-year interim results of a 2-year clinical trial in Chinese children. These initial myopia control results require further confirmation with the results obtained from the whole duration of the clinical trial. The generalisability of the results regarding the myopia control effect of the HAL and SAL lenses may be limited to Chinese children; thus, future trials in other ethnic populations are needed. Significant differences were observed in the gender distribution and average $\mathrm{AL}$ in the SAL group compared with the other two groups, despite participants' initial randomisation. Nevertheless, these differences did not significantly affect the study outcomes, as they had been adjusted in the multivariable analyses. Subjective methods such as phone interview and questionnaire are less reliable than objective measures. Future studies could employ wearable devices to measure wearing time and improve precision.

In summary, spectacle lenses with aspherical lenslets significantly reduced myopia progression and axial elongation in children. Among the treatment groups, the larger treatment effect was achieved by HAL. No treatment-related adverse events were reported, reflecting the comfort and safety of HAL and SAL for myopia control in children. If the full 2-year clinical trial results are consistent with the 1-year results, the use of HAL instead of conventional SVL for myopia correction would be a strategic approach to reduce myopia progression.

Contributors JB, $A Y$ and $H C$ designed the study. JB, YH, $X L, Y P$ and $C D$ performed the study. YH and EWL managed the data. JB, AY, JZ, DPS and BD analysed and interpreted the data. JB and $A Y$ wrote the initial draft. BD and $\mathrm{HC}$ revised the manuscript. FL and $\mathrm{HC}$ supervised the study. All authors provided a final review and approved the manuscript before submission.

Funding This work was supported by the International S\&T Cooperation Program of China (grant number 2014DFA30940) and the collaborative research project with Essilor International (Wenzhou Medical University grant numbers 95013006 and 95016010)

Competing interests Jinhua Bao is an Associate Director of Wenzhou Medical University-Essilor International Research Centre. Adeline Yang, Ee Woon Lim, Daniel P. Spiegel and Björn Drobe are employees of Essilor International. This company supplied the study devices and holds the following patent applications related to this work: WO2019166653, W02019166654, and W02019166655. No conflicts of interest exist for the remaining authors.

Patient consent for publication Not required.

Ethics approval The human ethics committees at the Eye Hospital of Wenzhou Medical University approved the protocol (Y2018-054). All protocols adhered to the tenets of the Declaration of Helsinki. All participants provided written informed consent.

Provenance and peer review Not commissioned; externally peer reviewed.
Data availability statement Data are available upon reasonable request.

Supplemental material This content has been supplied by the author(s). It has not been vetted by BMJ Publishing Group Limited (BMJ) and may not have been peer-reviewed. Any opinions or recommendations discussed are solely those of the author(s) and are not endorsed by BMJ. BMJ disclaims all liability and responsibility arising from any reliance placed on the content. Where the content includes any translated material, BMJ does not warrant the accuracy and reliability of the translations (including but not limited to local regulations, clinical guidelines, terminology, drug names and drug dosages), and is not responsible for any error and/or omissions arising from translation and adaptation or otherwise.

Open access This is an open access article distributed in accordance with the Creative Commons Attribution Non Commercial (CC BY-NC 4.0) license, which permits others to distribute, remix, adapt, build upon this work non-commercially, and license their derivative works on different terms, provided the original work is properly cited, appropriate credit is given, any changes made indicated, and the use is non-commercial. See: http://creativecommons.org/licenses/by-nc/4.0/.

\section{ORCID iDs}

Yingying Huang http://orcid.org/0000-0001-7694-513X

Fan Lu http://orcid.org/0000-0002-8794-6944

Hao Chen http://orcid.org/0000-0002-8594-5630

\section{REFERENCES}

1 Holden BA, Fricke TR, Wilson DA, et al. Global prevalence of myopia and high myopia and temporal trends from 2000 through 2050. Ophthalmology 2016;123:1036-42.

2 Naidoo KS, Fricke TR, Frick KD, et al. Potential lost productivity resulting from the global burden of myopia: systematic review, meta-analysis, and modeling Ophthalmology 2019;126:338-46.

3 Saw S-M, Gazzard G, Shih-Yen EC, et al. Myopia and associated pathological complications. Ophthal Physiol Opt 2005:25:381-91.

4 Wong Y-L, Sabanayagam C, Ding Y, et al. Prevalence, risk factors, and impact of myopic macular degeneration on visual impairment and functioning among adults in Singapore. Invest Ophthalmol Vis Sci 2018;59:4603-13.

5 Wildsoet CF, Chia A, Cho P, et al. Imi - interventions for controlling myopia onset and progression report. Invest Ophthalmol Vis Sci 2019;60:M106-31.

6 Smith EL, Hung LF. The role of optical defocus in regulating refractive development in infant monkeys. Vision Res 1999:39:1415-35.

7 Wallman J, Gottlieb MD, Rajaram V, et al. Local retinal regions control local eye growth and myopia. Science 1987;237:73-7.

8 Tse DY, Lam CS, Guggenheim JA, et al. Simultaneous Defocus integration during refractive development. Invest. Ophthalmol. Vis. Sci. 2007;48:5352-9.

9 Arumugam B, Hung L-F, To C-H, et al. The effects of the relative strength of simultaneous competing defocus signals on emmetropization in infant rhesus monkeys. Invest Ophthalmol Vis Sci 2016;57:3949-60.

10 Tse DY, To C-ho, To C. Graded competing regional myopic and hyperopic defocus produce summated emmetropization set points in chick. Invest Ophthalmol Vis Sci 2011;52:8056-62.

11 Woods J, Guthrie SE, Keir N, et al. Inhibition of defocus-induced myopia in chickens. Invest Ophthalmol Vis Sci 2013;54:2662-8.

12 Irving EL, Yakobchuk-Stanger C. Myopia progression control lens reverses induced myopia in chicks. Ophthal Physiol Opt 2017;37:576-84.

13 Berntsen DA, Barr CD, Mutti DO, et al. Peripheral defocus and myopia progression in myopic children randomly assigned to wear single vision and progressive addition lenses. Invest Ophthalmol Vis Sci 2013;54:5761-70.

14 Lam CSY, Tang WC, Tse DY-Y, et al. Defocus incorporated multiple segments (DIMS) spectacle lenses slow myopia progression: a 2-year randomised clinical trial. Br J Ophthalmol 2020;104:363-8.

15 Chamberlain P, Peixoto-de-Matos SC, Logan NS, et al. A 3-year randomized clinical trial of MiSight lenses for myopia control. Optom Vis Sci 2019;96:556-67.

16 Lam CSY, Tang WC, Tse DY-Y, et al. Defocus incorporated soft contact (disc) lens slows myopia progression in Hong Kong Chinese schoolchildren: a 2-year randomised clinical trial. Br J Ophthalmol 2014;98:40-5.

17 Chen $Y$, Jin W, Zheng Z, et al. Comparison of three monocular methods for measuring accommodative stimulus-response curves. Clin Exp Optom 2017;100:155-61.

18 Wang $Y$, Zhao K, Yang $X$, et al. Higher order aberrations and low contrast vision function in myopic eyes (-3.00 to -6.00 D) under mesopic conditions. J Refract Surg 2011;27:127-34

19 Yang Z, Lan W, Ge J, et al. The effectiveness of progressive addition lenses on the progression of myopia in Chinese children. Ophthal Physiol Opt 2009;29:41-8.

20 Olsen T. Sources of error in intraocular lens power calculation. J Cataract Refract Surg 1992;18:125-9.

21 Hasebe S, Jun J, Varnas SR. Myopia control with positively aspherized progressive addition lenses: a 2-year, multicenter, randomized, controlled trial. Invest Ophthalmol Vis Sci 2014; 55:7177-88

22 Tse DY, Lam CS, Guggenheim JA, et al. Simultaneous defocus integration during refractive development. Invest Ophthalmol Vis Sci 2007:48:5352-9. 


\section{Clinical science}

23 Berntsen DA, Sinnott LT, Mutti DO, et al. A randomized trial using progressive addition lenses to evaluate theories of myopia progression in children with a high lag of accommodation. Invest Ophthalmol Vis Sci 2012;53:640-9.

24 Chua SYL, Sabanayagam C, Cheung Y-B, et al. Age of onset of myopia predicts risk of high myopia in later childhood in myopic Singapore children. Ophthal Physiol Opt 2016;36:388-94.

25 Benavente-Perez A, Nour A, Troilo D. Short interruptions of imposed hyperopic Defocus earlier in treatment are more effective at preventing myopia development. Sci Rep 2019;9:11459.

26 Siegwart JT, Norton TT. Binocular lens treatment in tree shrews: effect of age and comparison of plus lens wear with recovery from minus lens-induced myopia. Exp Eye Res 2010;91:660-9.

27 Howlett MHC, McFadden SA. Spectacle lens compensation in the pigmented guinea pig. Vision Res 2009;49:219-27.

28 Read SA, Collins MJ, Sander BP. Human optical axial length and defocus. Invest Ophthalmol Vis Sci 2010;51:6262-9.
29 Chakraborty R, Read SA, Collins MJ. Monocular myopic defocus and daily changes in axial length and choroidal thickness of human eyes. Exp Eye Res 2012;103:47-54.

30 Nickla DL, Wildsoet C, Wallman J. Compensation for spectacle lenses involves changes in proteoglycan synthesis in both the sclera and choroid. Curr Eye Res 1997;16:320-6.

31 Troilo D, Nickla DL, Wildsoet CF. Choroidal thickness changes during altered eye growth and refractive state in a primate. Invest Ophthalmol Vis Sci 2000;41:1249-58.

32 Atchison DA. Optical models for human myopic eyes. Vision Res 2006;46:2236-50.

33 Sng CCA, Lin X-Y, Gazzard G, et al. Peripheral refraction and refractive error in Singapore Chinese children. Invest Ophthalmol Vis Sci 2011;52:1181-90.

34 Li S-M, Wang N, Zhou Y, et al. Paraxial schematic eye models for 7- and 14-year-old Chinese children. Invest Ophthalmol Vis Sci 2015;56:3577-83.

35 Chen X, Sankaridurg P, Donovan L, et al. Characteristics of peripheral refractive errors of myopic and non-myopic Chinese eyes. Vision Res 2010;50:31-5. 